\title{
The modelling of M-giant spectra
}

\author{
Bertrand Plez ${ }^{1}$ \\ Astronomiska observatoriet, box 515, S-75120 Uppsala and \\ Atomspektroskopi, Fysiska inst., box 118, S-221 00 Lund, Sweden
}

\begin{abstract}
I review the current status of cool O-rich giant star spectral modelling, stressing some specific problems like line profiles and molecular data completeness. I discuss recent progress in molecular line lists ( $\mathrm{TiO}, \mathrm{VO})$ and describe recent attempts in modelling normal M-giants as well as AGB star spectra, using static and hydrodynamic models. I anticipate further advances in the near future, with better radiative transfer in hydrodynamic models, that will allow detailed comparisons of models and observations, opening the possibility of deriving stellar parameters from spectroscopic and interferometric observations.
\end{abstract}

\section{Introduction}

The modelling of cool giant spectra has greatly developed and improved in recent years, as this volume witnesses, as a consequence, in particular, of the new window opened in the IR by ISO, and of the recent great increase in molecular line databases content and accuracy.

Some specific modelling attempts for late-type giant spectra, esp. Miras, are discussed in this volume, or in the posters published electronically at the ADS, by Aringer et al. (1999, ISO-SWS of LPVs), Decin et al. (1999, ISO-SWS of cool giants), Ryde et al. (1999, ISO-SWS of AGB stars), Schultheis et al. (1999, TiO bands of Miras and semi-regular variables), for O-rich objects, and by Aoki et al. (1999, ISO-SWS of carbon stars), Hron et al. (1999, ISO-SWS of carbon variables), Winters et al. (1999, IR light curves, center-to-limb variations and $\mathrm{CO}$ line profiles in carbon stars), for C-rich objects.

In this review I would like to focus on other specific recent results and emphasize some particular problems and difficulties encountered when modelling M-giant spectra. I will also show that models are now capable of reproducing observations to a level of accuracy that could not be achieved 1 or 2 years ago. Progress has been rapid indeed.

Synthetic spectra are absolutely necessary for the interpretation of observations and the extraction of fundamental data such as chemical abundances, effective temperatures, radii, etc. They also permit the conversion of theoretical models to observables, and thereby open the possibility of first testing the models, before using them to derive fundamental stellar quantities. One very fine example is provided by Winters et al. (1999), with their modelling of IR light

${ }^{1}$ present address: GRAAL, Université Montpellier II, 34095 Montpellier cedex 5, France 
curves and center-to-limb variations for the stars AFGL 3068 and IRC +10216. I do not think that the modelling has reached such a quality and self-consistency that we can unambiguously extract the luminosity, mass, log g, etc. of a particular star from comparisons of theoretical and observed light curves (the coupling between the pulsation driving zone and the atmosphere must first be achieved), but we can foresee a not so remote future where this might be possible.

The computation of spectra demands a knowledge of the thermal structure ( $\mathrm{T}$ and $\mathrm{P}$ vs. $\tau$ or $\mathrm{R}$ ) usually provided by a model, but which in extremely simplified modelling could be that of a single layer. Then, assuming or not chemical equilibrium and LTE, partial pressures of the various species can be computed, which combined with continuous and line opacities allow the calculation of a synthetic spectrum. The quality of the result largely reflects the adequacy to the real situation of the model, of the opacities and of the hypotheses made (LTE, geometry, staticity/homogeneity, ...). Currently, most modelling assumes LTE, chemical equilibrium, plane-parallel or spherical symmetry, and in a few cases velocity fields (see Windsteig et al. 1999; Winters et al. 1999).

\section{Intensity spectra}

It is convenient to separate the discussion into intensity and flux spectra. Intensity spectra provide us with the variation of the spectrum with the position on the stellar surface. They are the tool for understanding high-angular resolution observations, and to relate, for example, intensity distributions at various wavelengths to a "radius" at some reference Rosseland depth or pressure. It must be emphasized that interferometric observations do not provide "the" radius of the star, esp. not in the case of LPVs. Baschek et al. (1991) and Scholz (1997) have discussed the radius concept in detail, and I recommend reading these papers. The problem may be illustrated as follows: assume we wish to know the radius where $\tau_{R o s s}=1$, as it is the reference radius we use in our atmospheric or pulsation model (through, for example: $L=4 \pi R^{2} \sigma T_{\mathrm{eff}}^{4}$ and/or $g=G M / R^{2}$ ). Interferometric measurements will provide us with the center-to-limb variation of the intensity in one or more passbands centered on various wavelengths. These usually differ by large amounts, both in angular extension and in shape, in cool giants, and esp. in LPVs. It is thus impossible to derive $R\left(\tau_{R o s s}=1\right)$ purely empirically. A model must be used, that must reproduce the intensity profiles observed in all passbands, with a single set of $\left(L, T_{\text {eff }}, M, \ldots\right)$ parameters. This is all too rarely done.

One of the reasons is probably that few models exist and have been discussed in detail, although in principle the calculation of intensity profiles can be done for any published model atmosphere grid, if appropriate opacities are available. The only systematic computation has been published by Hofmann \& Scholz (1998) and by Hofmann et al. (1998), in their follow-up studies of the work of Bessell et al. (1996), for grids of static and hydrodynamic models of $\mathrm{M}$ giants and Miras. Their main conclusions are that: (i) it is necessary to use clean, well defined narrow passbands, that must avoid as much as possible the mixing of continuum and strong absorption bands. (ii) in Miras, the center-to-limb variations are very sensitive to details of the model; this makes the derivation of $R\left(\tau_{\text {Ross }}=1\right)$ very difficult, but on the other hand offers the possibility to 
efficiently test the models and put strong constraints on them. This, however, requires high-accuracy interferometric measurements (typically at the $1 \%$ level or better on visibilities, and past the first zero). I wish to warn here again that uniform-disk or even fully-darkened radii derived from visibilities are often a poor approximation of the real intensity profile of Mira stars, and that it is not trivial to derive, e.g., a "pulsation" radius from these, that may be used in P-R-L relations. There is most of the time little similarity between the "photometric" radius derived from observations and the "pulsation" radius used in theoretical modelling. The atmospheric model is crucial in establishing the link betwen them, provided it reflects reality. For normal M-giant center-to-limb variations, Hofmann et al. (1998a, b) conclude that in the IR fully-darkened diameters are a reasonably good approximation, and might be used, with caution, in the absence of better information on the brightness profile.

Important progress should arise in this field from detailed spectrum calculations with better up-to-date opacities. Observers and modellers must work together, carefully defining bandpasses and using good models and opacities. This will allow meaningful information to be extracted from high-angular resolution observations. See Lopez (1999), Monnier (1999), Irrgang et al. (1999), Karovska (1999), Käufl et al. (1999), Perrin et al. (1999), Tuthill et al. (1999), van Belle et al. (1999), and Weigelt et al. (1999) in these proceedings for further description of interferometric techniques and recent results.

\section{Flux spectra}

Flux spectra may be more or less arbitrarily divided into (very) low-spectral resolution $(R<\sim 1000)$ and (very) high-resolution $(R>\sim 20000)$. The latter is used for abundance analyses and for the study of circumstellar shells (velocity structure). Low resolution spectra allow the exploration of the spectrum for, e.g., the identification of molecular bands (see the various ISO SWS and LWS studies in this volume: e.g. Aoki et al. 1999, Hron et al. 1999, Cohen et al. 1999). Synthetic low-resolution spectra can be used for the derivation of broad-

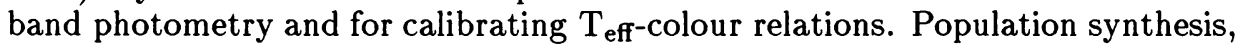
narrow-band colours and spectrophotometric work require intermediate resolutions. Some abundance analyses (like ${ }^{12} \mathrm{C} /{ }^{13} \mathrm{C}$ using the $\mathrm{CO}$ ro-vibration bands in the IR, see Plez et al. 1993) may be carried out at relatively low spectral resolution (a few 1000:s).

Estimates of the spectral energy distribution may be quickly calculated using mean or sampled opacities. This may lead to erroneous results, and whenever possible should be avoided. At least one control calculation should be done with a detailed line list at very high resolution (with a sampling much denser than the typical line width, i.e. $R>100000$ ). Large errors are especially likely if the line density is low. A coarse sampling may well systematically miss a large number of lines, leading to an underestimate of the opacity and thus an overestimate of the flux. The converse is also possible if mostly line centers are sampled. The problem is not very severe at high line densities, like for $\mathrm{TiO}$ in the optical, where all kinds of lines, -strong and weak-, will be sampled by even a relatively small number of wavelengths. 


\section{Potential problems}

When modelling the spectra of cool stars one is confronted with a number of questions and potential problems. The first is of course the adequacy of the model atmosphere being used: static or hydrodynamic, plane-parallel or spherical, with a variety of approximations (LTE, mixing-length theory for convection, etc.). I will not discuss this aspect which I developed elsewhere (Plez 1999). Other important issues are:

- the (in)completeness of line lists, missing oscillator strengths, uncertain line positions.

- the importance of pulsation, shocks and atmospheric extension.

- velocity fields, and line profiles.

- dust: present? under which form? opacities...

- circumstellar contributions.

- non homogeneity (spots, non-sphericity).

- non-LTE.

- non-equilibrium chemistry.

- ...?

Problems get more severe for Miras and tend to increase with decreasing $T_{\text {eff }}$. Let us very quickly mention a few of these problems before addressing the others in more detail.

Dust formation in O-rich stars is not well understood and most work on dust has been done in C-rich environments (cf. the reviews by Fleischer et al. 1999, Cherchneff \& Cau 1999, and Lodders \& Fegley 1999, and the works on O-rich dust formation of Jeong et al. 1999, and of Kosaza \& Sogawa 1999, in this volume). On the other hand, molecular opacities appear better known in O-rich stars. Alexander et al. (1999) attempt to model the impact on the emergent spectrum of the depletion of some atomic and molecular species due to dust formation.

Non-LTE has been very little explored, and only for specific lines (a few emission lines by Luttermoser \& Bowen 1992, or Li I by Kiselman \& Plez 1995). The impact of non-equilibrium chemistry on spectra remains unstudied.

I will not extensively discuss or provide detailed answers to all the items listed above, but rather draw attention to a few of them, and to recent progress and to newly obtained results. Discussion of some of the others will be found elsewhere in these proceedings (for example, Winters et al. 1999 show the effect of pulsation on the IR spectrum).

\subsection{Line profiles}

Let me first illustrate the problems with line profiles and with line list completeness through a few examples. In our spectroscopic study of galactic S-stars 
(Smith et al. in prep.) we found that the Li I $6707 \AA$ line profile is of complex structure and cannot be modelled with a simple hydrostatic model. This is obvious in $R=50000$ spectra of S Cas and other Miras. Moreover, the K I $7699 \AA$ resonance line displays a similar doubly-peaked profile. Other lines are likely to be alike. There could be a circumstellar contribution (both in absorption and emission, as observed around some stars by e.g. Mauron \& Caux 1992, Mauron 1997 and Plez \& Lambert 1994). Alternatively the profiles could be affected by velocity gradients in the extended (shocked) atmosphere. These hypotheses can be checked through time-monitoring of the profiles and through a comparison with higher excitation lines. Bessell et al. (1996) computed profiles for a few test lines in their hydrodynamic models for Mira. Their figures 13 through 15 nicely illustrate the possible importance of velocity fields in Mira atmospheres on their emergent spectra. Inspection of these figures readily shows that, as expected, even the equivalent width of the lines is affected compared to the hydrostatic conditions. Abundance analyses will be in error if not caring for this effect. More higher resolution spectra must be accumulated for a sample of LPV's and non-variable cool giants, and compared to static and hydrodynamic model spectra.

\subsection{Line lists completeness}

Another potential source of problem is the continuum placement. Early spectral abundance analyses of M giants (RGB or AGB) in the optical used the local continuum and synthetic spectra computed with line lists for $\mathrm{TiO}$ compiled from laboratory observations. Still worse, some analyses were based on equivalent widths. This resulted in inconsistent and systematically low abundances. Luck \& Lambert (1982) pointed out that this was probably due to unaccounted "pseudo-continuous" $\mathrm{TiO}$ absorption, which they modelled as a continuous opacity proportional to the $\mathrm{TiO}$ partial pressure. This was not very satisfactory but lead to much more coherent results. The use of a $\mathrm{TiO}$ line list computed up to much higher quantum numbers and with many more faint transitions included allowed us to account for this extra opacity and derive consistent abundances (Plez et al. 1993). In the case of a model typical for the MS stars at the tip of the Small Magellanic Cloud AGB $\left(\mathrm{T}_{\text {eff }}=3350 \mathrm{~K}\right)$ the local "continuum" around the $\mathrm{Li}$ I $6707 \AA$ doublet is at $30 \%$ of the true continuum which is not reached anywhere near in the spectrum. Two thirds of that depression are due to $\mathrm{TiO}$ lines which are unobserved in the laboratory... The key here is to use as complete a molecular line list as possible, and use lines of the same element from very different spectral regions thus differently affected by molecular blanketing. It is thus crucial to use the subordinate $8126 \AA$ line (and $6103 \AA$ when possible) in conjunction with the $6707 \AA$ doublet when deriving Li abundances in cool giants.

To conclude, although abundances have been, and are derived, that seem consistent and mostly follow our expectations in terms of nucleosynthesis and late stellar evolution, there is a need to check line profiles at higher spectral resolution ( 2 to $3 \mathrm{~km} / \mathrm{s}$ ) in Miras or irregular variables against non-variable stars for various types of spectral lines. Some test analyses could be conducted with hydrodynamic model atmospheres, including velocity fields, and compared to similar analyses done with hydrostatic models. 


\section{Recent results and progress}

Despite, or maybe because of, these problems and others not discussed here, tremendous progress was achieved in the past few years. The development of atmospheric modelling is reviewed by Plez (1999) and by Höfner (1999) in this volume.

\subsection{Molecular line lists}

Some persistent questions concerning opacity sources have been solved very recently. To cite a few examples:

- VO: Karlsson et al. (1997) measured the lifetime of the three excited A, $\mathrm{B}$ and $\mathrm{C}$ states, providing the opportunity to calculate for the first time laboratory gf-values to replace the (not so good) astrophysical calibrations.

- TiO: The ab initio calculations of Langhoff (1997) are in good agreement with the laboratory determinations of Hedgecock et al. (1995). New line lists were recently published (Plez 1998; Schwenke 1998). Plez carried out detailed comparisons of his line list with laboratory data and M-giant spectra. The match with observations is good, with the exception, however, of the $\delta$ band for which the strength from the Langhoff calculations is too large in M-giants. I recommend laboratory determination of the lifetimes and the branching ratios of the singlet states, which unfortunately seem not easy to obtain accurately.

- $\mathrm{H}_{2} \mathrm{O}$ : a new ab initio line list was recently proposed by Partridge \& Schwenke (1997), which seems of good quality, but which has not yet been extensively tested in M-giant (and dwarf) model atmospheres.

However, for the modelling of very cool objects, and for S-stars, some metal-oxide opacities are still missing. E.g. for $\mathrm{LaO}$, there exists no lifetime measurement of the excited A levels, and gf-values for the A-X transition (bands around 7400 and $7900 \AA$ ) can only be guessed.

\subsection{Some illustrations}

The leap forward in computing power and storage capabilities of the last 3-4 years means that very extensive line lists can be used in the modelling of stellar atmospheres and spectra. Large portions of spectra, like the ISO SWS range, or the whole optical spectrum, may now routinely be computed for a grid of models, at high-resolution, and including tens of millions of spectral lines, without the recourse to a CRAY. Beautiful results have emerged recently as a consequence of this ( $\mathrm{r}$ )evolution.

Bessell et al. (1998) have shown that good UBVRIJHK Cousins-Johnson photometry can now be derived for K-M stars from modern model grids (the Uppsala recent grids of MARCS models) incorporating up-to-date opacities. Plez (1998) demonstrated that the optical spectrum of M0 to M5 III stars is well modelled with his $\mathrm{TiO}$ line list (see his figure 2). Another nice piece of work is that of Alvarez \& Plez (1998). Their pioneering attempt to model Lockwood (1972) narrow-band colour observations of O-rich Miras opens the prospect of 
detailed testing of hydrodynamic models against observations. Lockwood (1972) observed a total of 256 Miras at various phases of their cycle through 5 photometric bands of about $50 \AA$ width between 0.75 and $1.1 \mu \mathrm{m}$, sampling some TiO and VO absorption bands. He noted that Miras describe loops in colour-colour diagrams during their cycle. This can be qualitatively understood by the running of a shock (or some perturbation) through the extended atmosphere. As different $\mathrm{TiO}$ and $\mathrm{VO}$ absorption bands form at different depths they are affected at different times, and phase lags appear between the "TiO" and "VO" colours (the average phase lag is about 0.1 here). Alvarez \& Plez demonstrated that a sequence of current static MARCS model atmospheres and the new updated $\mathrm{TiO}$ and $\mathrm{VO}$ line lists reproduce the colours of ordinary M-giants observed by Lockwood. Thereafter they computed LTE radiative transfer in a time-series of hydrodynamical models for a typical LPV (from Höfner, priv. comm.) using the same line lists. Despite the limitations (LTE, one single model compared to an observed star defined by the average of 256 observed stars, no velocity fields taken into account in the radiative transfer ,...), the model colour variations are in surprisingly good agreement with the observations. Both the amplitude of variation and the phase-lag are reproduced. The absolute values of some of the model colours are however too large. We are still far from deriving stellar parameters from such modelling, but we are certainly getting closer to it. Such detailed modelling and comparison to high-quality observations (low- and higher-resolution spectra, narrow band colours) will allow extensive testing of the models. Also the modelling should include velocity fields, which will affect the spectrum, as shown by Bessell et al. (1996) and Windsteig et al. (1999).

These progresses open new perspectives in the modelling of M-giant spectra, and of cool stars in general, including LPVs. Note, however, that due to larger uncertainties in the existing opacities of carbon-bearing polyatomic molecules, as well as the yet unknown absorption sources of potential importance (e.g. $\mathrm{C}_{2} \mathrm{H}$ ), the modelling of carbon star photospheric spectra lags slightly behind. Although the work of Hron and his collaborators $(1998,1999)$ may well demonstrate that this is quickly changing.

\section{Conclusions}

We are now able to reproduce the spectra of normal M-giants (although whether the center-to-limb variations can be properly modelled remains to be seen through detailed comparisons with interferometric data). Attempts to model LPV spectra with currently affordable methods (i.e. a posteriori LTE radiative transfer in a temperature-density atmospheric structure computed with a hydro-code) have been successful beyond expectations. Some discrepancies remain, and it is certainly fundamental for our understanding to find out where they stem from, but the road is open to a quantitative use of the models to accurately model stellar populations or convert theoretical evolutionary sequences $\left(\mathrm{T}_{\text {eff- }}-\mathrm{L}\right)$ into observed H-R diagrams (colour-magnitude). And, maybe of more interest, we are at the beginning of this exciting new era where we finally are able to test theoretical hydrodynamic models of LPVs against observations of light curves, colours, spectra and center-to-limb variations. This will bring us to an in-depth understanding of (pulsating) AGB stars through, of course, the accurate deter- 
mination of $\mathrm{T}_{\text {eff }}, \mathrm{R}$, abundances, $\mathrm{M}$, etc., but also, of the physical mechanisms at work in these fascinating objects, their interactions (e.g. between the pulsation driving zone and the atmospheric filter) and their consequences (e.g.: why is the phase shift between $\mathrm{TiO}$ and VO colours about 0.1 ? This must reflect some general scaling property between the atmosphere's size, the velocity of the shock waves, the pulsation period, etc.). If we add to this the fact that hydrodynamic models will soon be computed with a consistent treatment of the radiation field, and that model spectra will include the effect of velocity fields and, at least partly, of non-LTE, we can bet on a rich harvest of results for the next AGB symposium!

Acknowledgments. Bengt Gustafsson is thanked for financial support during the course of this work. I thank S. Höfner, K.H. Hofmann, M. Scholz and T. Tsuji for sending (p)reprints. Financial support was provided by the Swedish NFR.

\section{References}

Alexander D.R., Ferguson J.W., Wing R.F., Johnson H.R., Haushchildt P.H., Allard F., 1999, this volume

Alvarez R., Plez B., 1998, A\&A 330, 1109

Aoki W., Tsuji T., Ohnaka K., 1999, this volume

Aringer B., Kerschbaum F., Hron J., Posch T., Windsteig W., Jørgensen U.J., Höfner S., 1999, this volume

van Belle G., The PTI collaboration, Thomson R.R., 1999, poster contribution, I.A.U. Symposium No. 191

Baschek B., Scholz M., Wehrse R., 1991, A\&A 246, 374

Bessell M.S., Castelli F., Plez B., 1998, A\&A 333, 231 (erratum A\&A 337, 321)

Bessell M.S., Scholz M., Wood P.R., 1996, A\&A 307, 481

Cherchneff I., Cau P., 1999, this volume

Cohen M., Barlow M.J., Sylvester R.J., Liu X.-W., Cox P., Lim T., Schmitt B., Speck A.K., 1999, this volume

Decin L., Cohen M., Eriksson K., Gustafsson B., Huygen E., Morris P., Plez B., Sauval J., Vandenbussche B., Waelkens C., 1999, poster contribution, I.A.U. Symposium No. 191

Fleischer A.J., Winters J.M., Sedlmayr E., 1999, this volume

Hedgecock I.M., Naulin C., Costes M., 1995, A\&A 304, 667

Hofmann K.H., Scholz M. 1998, A\&A 335, 637

Hofmann K.H., Scholz M., Wood P.R., 1998, A\&A 339, 846

Hron J., Loidl R., Höfner S., Jørgensen U.J., Aringer B., Kerschbaum F., Windsteig W. 1998, A\&A 335, L69

Hron J., Loidl R., Höfner S., Jørgensen U.G., Aringer B., Kerschbaum F., Windsteig W., 1999, this volume

Höfner S., 1999, this volume 
Irrgang P., Balega Y.Y., Gauger A., Osterbart R., Schniggenberg G., Weigelt

G., 1999, poster contribution, I.A.U. Symposium No. 191

Jeong K.S., Winters J.M., Sedlmayr E. 1999, this volume

Karlsson L., Lindgren B., Lundevall C., Sassenberg U., 1997, J. Mol. Spectroscopy 181,274

Karovska M., 1999, this volume

Kiselman D., Plez B., 1995, Mem. Soc. Ast. It. 66, 429

Kozasa T., Sogawa H., 1999, this volume

Käufl H.U., Stecklum B., Richichi A., Righter S., 1999, poster contribution, I.A.U. Symposium No. 191

Langhoff S., 1997, ApJ 481, 1007

Lockwood G.W., 1972, ApJS 24, 375

Lodders K., Fegley B., Jr., 1999, this volume

Lopez B., 1999, this volume

Luck R.E., Lambert D.L., 1982, ApJ 256, 189

Luttermoser D.G., Bowen G.H. 1992, in CSSS, 7th Cambridge workshop, ASP

Conf. Series 26, 558

Mauron N., 1997, A\&A 326, 300

Mauron N., Caux E., 1992, A\&A 265, 711

Monnier J.D., 1999, this volume

Partridge H., Schwenke D.W., 1997 J. Chem. Phys. 106, 4618

Perrin G., Coudé du Foresto V., Ridgway S.T., Mariotti J.-M., Ruilier C., Mennesson B., Traub W.A., 1999, poster contribution, I.A.U. Symposium No. 191

Plez B., 1998, A\&A 337, 495

Plez B., 1999, in "The C-star Phenomenon", ed. R.E. Wing, Kluwer

Plez B., Lambert D.L., 1994, ApJ 425, L101

Plez B., Smith V.V., Lambert D.L., 1993, ApJ 418, 812

Ryde N., Eriksson K., Gustafsson B., Olofsson H., Plez B., 1999, poster contribution, I.A.U. Symposium No. 191

Scholz M., 1997, in Fundamental Stellar Properties, eds. T.R. Bedding et al.

Schultheis M., Aringer B., Höfner S., Jørgensen U.J., 1999, poster contribution, I.A.U. Symposium No. 191

Schwenke D.W., 1998, Faraday discussion 109: Chemistry of molecules and grains in space, in press

Smith V.V., Plez B., Lambert D.L., et al., in preparation

Tuthill P.G., Monnier J.D., Danchi W.C., 1999, this volume

Weigelt G., Blöcker T., Hofmann K.H., Osterbart R., Balega Y.Y., Fleischer A.J., Winters J.M., 1999, this volume

Windsteig W., Aringer B., Lebzelter T., Höfner S., 1999, poster contribution, I.A.U. Symposium No. 191

Winters J.M., Le Bertre T., Keady J.J., 1999, this volume 